\title{
El contexto de la Covid-19 como espacio para repensar la virtualización educativa por parte de docentes universitarios
}

\section{The context of Covid-19 as a space to rethink educational virtualization in university teachers}

\section{Gerson Alexander Olivares-Parada \\ Universidad de Pamplona, Cúcuta, Colombia \\ Paola Olivares-Parada \\ Universidad Francisco de Paula Santander, Cúcuta, Colombia \\ Doris Parada-Rico \\ Universidad Francisco de Paula Santander, Cúcuta, Colombia}

\section{Resumen}

Objetivo: Determinar los cambios en las actitudes, usos e intereses de formación en las tecnologías de la información y la comunicación por parte de docentes universitarios durante la Covid-19. Métodos: Estudio cuantitativo, descriptivo, longitudinal, comparativo con 154 docentes de la Facultad de Ciencias de la Salud de una universidad pública, a quienes se les había aplicado el mismo instrumento en el año 2017. La encuesta utilizada "actitudes, usos e intereses de formación de los docentes en las TIC" posee validez de contenido, criterio y constructo, así como confiabilidad interna global de 0,94. Resultados: Debido a la patología emergente, se adopta la modalidad virtual exclusiva en el ámbito educativo, como herramienta privilegiada. El espacio social de interacción virtual y el uso de las tecnologías es asumido por el $82 \%$ de los docentes con actitud positiva, se avanza en la profundización y experticia para el manejo de recursos web $2.0(71,1 \%)$, se da participación ingente $(97 \%)$ en módulos de aprendizaje sobre Tecnologías de Información y Comunicación. Conclusiones y discusiones: A diferencia del periodo anteriormente evaluado (2017), se develan cambios en la subjetividad y accionar docente entramados entre el acontecimiento pandémico, la normativa estatal, sus presaberes y las capacitaciones realizadas, que confluyen en la construcción de prácticas pedagógicas virtualizadas.

Palabras clave: Comunicación, comportamiento social, docente, tecnología de la información, formación.

\begin{abstract}
Objective: To determine the changes in the attitudes, uses and interests of training in information and communication technologies of university teachers during the Covid-19. Method: Comparative longitudinal descriptive quantitative study with 154 teachers from the faculty of health in a public university, to whom the same instrument had been applied in 2017. The survey used "attitudes, uses and training interests of teachers in the TIC "has content, criterion and construct validity, as well as global internal reliability of 0,94 . Results: Due to the emerging pathology, the exclusive virtual modality is adopted for the educational field; As a privileged tool, the social space of virtual interaction and the use of technologies is assumed by $82 \%$ of teachers with a positive attitude, progress is made in deepening and expertise for the management of web 2.0 resources $(71.1 \%)$, There is huge participation (97\%) in learning modules on Information and Communication Technologies. Discussion and conclusions: Unlike the period previously evaluated, changes in subjectivity and teaching actions are revealed, intertwined between the pandemic event, state regulations, their pre-knowledge and the training carried out, which converges in the construction of virtualized pedagogical practices.
\end{abstract}

Open Access:

ISSN: $0124-2121$ E-ISSN: $2665-2420$

Editor:

Dhayana Fernández Matos

\author{
ARTÍCULO DE IN̦VESTIGACIÓN \\ CIENTÍFICA \\ Copyright @ \\ By Educación y Humanismo
}

Gerson Olivares gerson.olivares2@unip maplona.edu.co

Recibido: $29-08-2020$ Aceptado: $25-11-2020$ En línea desde: $10-02-2020$

Keywords: Communication, social behavior, teaching, information technology, training 


\section{Introducción}

Si bien el inicio del uso de las tecnologías de información y comunicación (TIC) como la radio y televisión en Colombia tuvo un impacto contundente durante el siglo pasado, fue la implementación de equipos de computación e internet, los que iniciaron un gran cambio en la sociedad (Ávila, 2013).

No obstante, en el ámbito educativo, las actitudes de los docentes ante la adopción de estos medios, sus usos e intereses de formación, no habían sido tan notorias como las que se han dado en la Covid-19. Esto implicó, de manera repentina, de construir subjetividades en torno a la aplicación de las tecnologías de información y comunicación en el desarrollo de los cursos y transformar el quehacer docente alineado, ahora, a la virtualización.

Este evento inesperado, declarado como una pandemia por la Organización Mundial de la Salud (OMS) en marzo del presente año (Ministerio de Salud y Protección Social, 2020), ocasiona la emergencia de salud pública más relevante de los últimos años, con tan altos riesgos para la vida humana que el Comité de Emergencia del Reglamento Sanitario Internacional (RSI) determina la necesidad de confinamiento social obligatorio (OPS-OMS, 2020).

Lo anterior, conllevó a que, en Colombia, la Presidencia de la República, según Decreto 417 del 17 de marzo de 2020, declarara el país en Estado de Emergencia Económica, Social y Ecológica $\mathrm{y}$, posteriormente, se emitieran normativas que dispusieran el teletrabajo, trabajo en casa y la virtualización educativa como medidas trascendentales para contener la propagación de la enfermedad.

En ese sentido, las TIC emergen como una herramienta vital para dar continuidad a los procesos educativos y permitir la interacción estudiante-docente. Tal como se configura por algunos autores, propician una serie de transformaciones en la humanidad y en sus diversas prácticas (Castells, 2008), en donde la "sociedad red" realiza sus acciones apoyada por las TIC y vivencia nuevos tiempos marcados por acontecimientos que transforman el paisaje social (Castells, 1996).

En ese orden de ideas, en el presente artículo damos cuenta de la pertinencia de las TIC en la educación y su inclusión en la normatividad colombiana. Se expone, en un segundo aparte, el fundamento teórico y estado del arte respecto a las actitudes, los usos e intereses de formación de los docentes en las TIC. El tercero, se refiere a los ajustes en la modalidad educativa dados a partir de la pandemia por Covid19 y, finalmente, se presentan los hallazgos de la investigación realizada en el municipio de Cúcuta con docentes de la Facultad de Ciencias de la Salud de una universidad pública de la región durante el periodo actual de pandemia, comparando estos resultados con los hallados en el periodo 2017, anteriormente evaluado. 


\section{Alineación de las TIC en la normatividad colombiana}

Se considera que a partir de las posibilidades tanto instrumentales como informacionales que se tengan en el contexto de la virtualidad, y de la apertura que los sujetos den al uso de herramientas virtuales, pueden ajustarse las dinámicas económicas, formas de comunicación, las políticas, el sistema educativo, entre otros, de una sociedad; lo cual implica a su vez, la ocurrencia de desarrollos y ajustes en las diferentes regiones (Katz, 2009; Salazar, 2015; Cázares y Urbiola, 2015).

Colombia, a partir de la Ley 1341 del 30 de julio de 2009, impulsa el uso de las TIC, denominándolas como "el conjunto de recursos, herramientas, equipos, programas informáticos, aplicaciones, redes y medios que permiten la compilación, procesamiento, almacenamiento y transmisión de información, como voz, datos, texto, vídeo e imágenes" (Congreso de la República de Colombia, 2009, artículo 6); igualmente se propone a partir de su uso el incremento de la productividad y la competitividad basado en un enfoque de derechos e inclusión social.

En consecuencia, para el sistema educativo, a partir de estas tecnologías (TIC) se pretende aportar a la calidad en la educación y romper con esquemas tradicionales unidireccionales, innovando vías pedagógicas que enriquecen el proceso de enseñanza y aprendizaje (Lucumí y González, 2015), lo cual se asume en el Plan Nacional de Educación vigente (2016-2026), que establece el "uso pertinente, pedagógico y generalizado de las nuevas y diversas tecnologías para apoyar la enseñanza, la construcción de conocimiento, el aprendizaje, la investigación y la innovación" (MEN, 2017, p. 17).

Así las cosas, a partir de la identificación de las necesidades del contexto y retos de la sociedad digital, se ha dado relevancia al planteamiento de "formar a los maestros en el uso pedagógico de las diversas tecnologías" (MEN, 2017) y se encaminan los proyectos al diseño de programas para el equipamiento de aulas y la formación de docentes y estudiantes en este ámbito, evidenciando que la formación de profesores en las TIC, incide, por ejemplo, en el aumento del puntaje de las pruebas Saber 11 en los estudiantes en 10,6 \% y así mismo, en el paso de estos a la educación superior (Departamento Nacional de Planeación, 2020).

Siguiendo esta evidencia, a nivel nacional el Ministerio de Educación ha dado apertura a programas como: "Aprender Digital", en busca de la transformación interactiva, lo cual a su vez implica grandes esfuerzos, de una parte, cambio en las formas de aprendizaje de los estudiantes y de otra, transformación de las prácticas pedagógicas y estrategias didácticas por parte de los docentes (Departamento Nacional de Planeación, 2020).

Es importante destacar que, aun cuando se proponen programas y estrategias con el propósito de mantener una educación de calidad con el apoyo de las TIC, se evidencian grandes brechas entre el ideal planteado y sus logros, especialmente con marcadas diferencias entre el área urbana y rural (Sánchez y Robles, 2016). Sirva de ejemplo los datos para el año 2016, según los cuales el $65,4 \%$ de la población urbana utilizaba internet como una herramienta de conectividad, 
mientras que sólo el 28,7\% de la rural lo hacía (Departamento Nacional de Planeación, 2018). Lo anterior relacionado con la falta de electricidad e internet en el área rural (Departamento Nacional de Planeación, 2020), lo cual afecta su uso.

\section{Las actitudes, usos e intereses de formación en las TIC en docentes universitarios}

Con respecto a la actitud hacia el uso de las TIC, puede definirse como una disposición del sujeto para aplicar estas tecnologías en su cotidianidad. No obstante, esta actitud se relaciona con nociones preconcebidas, temores, convicciones, entre otros, que pueden afectar la toma de decisiones para su uso, y en el caso de los docentes, para aplicarlas en sus prácticas pedagógicas (Sánchez, García, Steffens y Hernández, 2019). Igualmente, las actitudes son dinámicas, varían y pueden ser transformadas según experiencias y contextos (Herdina \& Jessner, 2002; Álvarez et al., 2011).

Entre los elementos que subyacen a la adopción de las tecnologías en el contexto educativo, Tapia (2018) refiere que se integran las creencias, los conocimientos y habilidades en su aplicación, lo cual puede fomentar o inhibir su uso. Así mismo, se identifica que, dentro del componente pedagógico de la actitud hacia las TIC, se hallan tres elementos importantes que son: el afectivo, comportamental y cognitivo (Tobón, Arbeláez, Falcón y Bedoya, 2010), los cuales dan cuenta de la disposición que tiene el sujeto para el uso de estas herramientas y artefactos.

Desde otro ángulo, se evidencia que el uso de las TIC puede darse en tres niveles de acuerdo con el alcance de competencias de los docentes en el manejo de estas. Según la Organización de las Naciones Unidas para la Educación, la Ciencia y la Cultura (Unesco, 2019), estos pueden clasificarse en Nivel I, relacionado con la adquisición de conocimientos y el uso de herramientas de productividad, procesamiento gráfico, presentaciones multimedia; Nivel II, profundización del conocimiento, en el que se utilizan herramientas Web 2 con mayor grado de facilidad en su aplicación; y un Nivel III, de creación de conocimiento.

Por consiguiente, es un engranaje entre las actitudes favorables de los docentes, la experticia e intereses de formación de estos para el uso de las TIC en sus prácticas pedagógicas, pero además, una intersección con las realidades del contexto que facilitan o no, llevar a cabo una inclusión de estas de manera coherente en el currículo, teniendo en cuenta la propuesta pedagógica y las particularidades del contexto de formación (Cariaga, 2018).

Por tal razón, se han realizado estudios en torno a las TIC, hallando, respecto a las actitudes de los docentes, dos perspectivas: una que hace referencia a la actitud negativa para su uso en las prácticas pedagógicas, en razón a la creencia de la deshumanización, el plagio, la pérdida de la interacción presencial y ausencia de tiempo para organizar y preparar contenidos (Echeverri, 2014; García, Ulloa y Córdoba, 2020; Vesga y Vesga, 2012; Dussel, 2015; Araya y Orellana, 2018).

Otra, que connota una actitud de proactividad en búsqueda del uso de las TIC como apoyo docente, por lo que se propicia la formación en ellas en busca de la coherencia entre la tecnología 
y una buena práctica pedagógica (Ehuletche, Lado, Atlante y Malbernat, 2018; Tello y Cascales, 2015; Rivera, Fernández, Guzmán y Pulido, 2017; Colina y Túa, 2018; Lucumí y González, 2015). Así mismo, se valora en algunos casos, la ambigüedad que existe entre las actitudes, los usos y deseos de formación en el ámbito de las TIC, puesto que se identifica en los docentes la importancia que otorgan a las TIC en las prácticas pedagógicas, pero no se observa un uso adecuado en las mismas (Tobón et ál., 2010; Padilla, 2018).

A nivel nacional e internacional, se hallan investigaciones que evidencian una imbricación entre elementos tanto de tipo instrumental relacionadas con las necesidades del contexto para hacer posible la interactividad virtual, así como del sujeto, de tipo comportamentales y cognitivas, en relación al déficit de conocimientos para el uso de las TIC y dificultades para entretejer las dimensiones disciplinar, pedagógica y tecnológica en el proceso de enseñanza y aprendizaje en torno a la virtualidad (Said, 2012; Cabero, 2015; Tello y Cascales, 2015; Cejas y Navío, 2020; Cariaga, 2018; Tejada y Pozos, 2018).

Además, en relación con los intereses de formación, se halla que los docentes desean capacitarse en este tema, y que de acuerdo con su nivel de experticia, proponen continuar en niveles de profundización y creación de conocimiento, como componentes del sistema educativo TIC (Unesco, 2019).

\section{Contexto educativo en la pandemia por SARS 2- Covid 19}

En medio de la complejidad que en sí misma encierra la práctica pedagógica a través del uso de las TIC, en la cual el docente orienta la construcción de conocimiento, intentando que el estudiante logre vivenciar una experiencia educativa que impacte y trascienda los límites del espacio virtual, emerge en el contexto mundial actual, un evento de salud pública que suscita cambios en todos los ámbitos de la vida cotidiana, incluyendo la educación, y que obliga en algunos casos a pasar de la modalidad netamente presencial a la virtualización educativa exclusiva, y a deconstruir tanto actitudes, como usos y aprendizajes en torno a las TIC: la pandemia por SARS 2- Covid 19.

Esta situación llevó a que diversos países replantearan los procesos académicos en las instituciones educativas y diseñaran o relevaran el uso de plataformas virtuales para continuar el desarrollo de clases en la modalidad a distancia. Por ejemplo, Chile diseñó el Plan de Acción por Coronavirus; Venezuela, el Plan Pedagógico de Orientación y Prevención; Costa Rica emitió las orientaciones para el Apoyo del Proceso Educativo a Distancia; Panamá estableció la estrategia "La Estrella de la Educación no se detiene"; Paraguay conformó el Consenso Educativo en el marco del "Plan de Educación en tiempos de Pandemia", y en el caso de Colombia, se propuso la formación virtual gratuita apoyada por entidades universitarias de ámbito privado y soportado en la plataforma web "Aprender Digital" (Unesco, 2020).

Así, Colombia estableció el "Plan Padrino" con el fin de promover la formación docente y el desarrollo de habilidades en las TIC interinstitucionalmente, de tal manera que, a partir del 
intercambio de experiencias pedagógicas, se apoya la continuidad de los procesos académicos de enseñanza y aprendizaje en los espacios virtuales. De esta forma, y en aras de fomentar la formación del docente universitario en este tema y dar paso a la virtualidad educativa como una experiencia pedagógica exitosa, la universidad pública del presente estudio, se alió con una universidad privada colombiana.

Cabe resaltar que el desafío para el sistema educativo, no sólo radica en transformar las prácticas pedagógicas, o la modalidad presencial, si no también supone revisar entre otros, la forma de asumir la virtualidad, los usos e intereses de formación en el área por parte de los docentes, como la apropiación que hacen de ellos. Por esta razón se lleva a cabo el presente estudio con el objetivo de identificar las diferencias en estos aspectos entre los periodos de medición 2017 y 2020 de los docentes universitarios referidos anteriormente.

\section{Método}

Para la realización de la presente investigación se planteó un estudio con enfoque cuantitativo que permitió recoger datos numéricos para medir patrones actitudinales y de comportamiento. El método usado es el descriptivo longitudinal comparativo, puesto que conduce a esbozar las características de situaciones y relacionar variables entre periodos.

La población objeto de estudio estuvo conformada por 154 docentes de la Facultad Ciencias de la Salud ( $98 \%$ del total), quienes facilitaban el desarrollo de los cursos en el periodo inicial del aislamiento social obligatorio decretado a nivel nacional, aceptaron hacer parte del estudio, y diligenciaron la encuesta enviada a través de formularios de Google o de grupos de WhatsApp, lo cual se realizó con previa autorización de las directivas de la facultad. El muestreo es de tipo probabilístico y fue llevado a cabo durante los meses de abril y mayo de 2020.

Se tuvo como criterios de inclusión que fueran docentes de la Facultad de Ciencias de la Salud, que estuviesen contratados en el actual semestre y que hubiesen participado en la anterior investigación realizada en el año 2017. Se aplicó el instrumento denominado "Actitudes, usos e intereses de formación docente con respecto a las TIC en la educación", diseñado por autores de la Universidad Tecnológica de Pereira en el año 2010 (Tobón et al„, 2010). Este fue autorizado para su uso y reproducción en actividades de tipo académicas y/o educativas.

Además, este instrumento cuenta con validez del contenido, de criterio y del constructo, que permiten evidenciar a su vez una medición de la fiabilidad interna de 0,9438, a partir del coeficiente Alpha de Cronbach para ítems con respuesta en escalas de grado, y el coeficiente de Kuder y Richardson KR -20 para ítems con respuesta dicotómica. Consta de nueve (9) preguntas abiertas, de información general y 68 cerradas en escalas tipo Likert, clasificadas de la siguiente manera:

Un primer subgrupo de 36 preguntas de tipo cerrado, que miden las actitudes con respecto a 
las TIC, y permiten la valoración entre cinco ítems que van desde muy de acuerdo a muy en desacuerdo, calificados así mismo de 1 a 5 respectivamente, pudiendo obtener de manera global en esta categoría un valor máximo de 180 y mínimo de 36. Esta categoría de actitud, a su vez está integrada, siguiendo a Estrada (2002), citada por Tobón et., ál (2010), por las subcategorías afectivo, comportamental y cognitivo, lo cual permite discriminar entre sí.

Un segundo subgrupo de 8 preguntas cerradas en escala de Likert, que evalúan la frecuencia de uso e interés de aprendizaje por las tecnologías de información, las cuales se distribuyen teniendo en cuenta los componentes del sistema educativo TIC establecido por la Unesco (2019). El uso de estas, se valoran entre 0 a 5 , en donde 0 , se relaciona con "no lo conozco" y 5 con la frecuencia de uso "siempre". El interés de aprendizaje va de 0 a 2, en una escala que transita entre nada interesado a muy interesado.

Tiene un tercer subgrupo de 12 preguntas que hacen referencia a la experticia que tienen los docentes en las tecnologías de la comunicación y el interés de aprendizaje en ellas. Estas califican el nivel de experticia entre 0: "no conozco", 1: básico, 2: intermedio y 3, nivel avanzado. El interés de aprendizaje se califica igual que la categoría anterior.

Contiene un cuarto subgrupo de 12 preguntas que revela el interés de formación de los docentes en los componentes del sistema educativo TIC, según enfoque propuestos por la Unesco (Nociones básicas, profundización de conocimiento y creación de conocimiento). Su valoración puede asignarse entre 1 y 3, según el grado de interés en los componentes, indicando 1, falta total de interés, 2, estar algo interesado y 3 poseer mucho interés en su aprendizaje.

Finalmente, tiene un grupo de 18 preguntas que hacen referencia a los obstáculos percibidos para el uso y apropiación de las TIC en la educación. Este se valora en respuestas dicotómicas según se presente o no la característica.

En cada categoría de preguntas, se otorga un valor máximo y mínimo según la escala valorativa y el número de preguntas de cada una, lo cual permite, a su vez, identificar de manera global la apreciación obtenida.

Para realizar la aplicación del estudio y diligenciamiento de encuestas, se tuvo en cuenta los principios éticos dados en la declaración de CIOMS, así como los referentes en la Resolución 8430 de 1993, donde se establecen las normas académicas y administrativas para la investigación en seres humanos.

\section{Resultados}

Con respecto a la información general, se obtuvo que la edad de los docentes se encuentra en rango de 26 a 73 años, sólo el $14 \%$ es masculino y el restante femenino. Su experiencia educativa se encuentra entre los 3 a 45 años de docencia universitaria. Entre sus profesiones se encuentran la enfermería (66\%) con niveles de postgrado en rangos de especialista a doctorados; $16 \%$ 
profesionales en fisioterapia, ingeniería industrial y psicología con nivel de especialidad y maestrías, y el porcentaje restante, distribuido entre médicos (2\%), químicos farmacéuticos $(4 \%)$, ingenieros industriales (8\%) y administradores de servicios de salud (4\%) con diversos niveles de postgrados.

Respecto a su contratación, el 68\% es catedrático, lo cual hace referencia al grupo docente que recibe el pago de nómina según horas contratadas; planta (18\%), vinculado como servidor público o de planta; el $9 \%$ es ocasional, quienes laboran con contratos acordes a su nivel de estudios con término definido por un año, y finalmente, el grupo de tutores que ocupan un $5 \%$ y laboran generalmente en programas a distancia.

\section{Actitudes con respecto a las TIC}

En esta categoría, se evaluaron los tres componentes pedagógicos de la actitud: cognitivo, afectivo y conductual. De esta forma, se identifica con respecto al componente cognitivo, que los docentes tienen una actitud positiva frente al uso de las TIC; consideran que estas son importantes para enriquecer el proceso de enseñanza y aprendizaje, así como para su crecimiento personal. Se obtiene en promedio un valor de 45, el cual está inserto entre el rango muy de acuerdo y de acuerdo.

Este valor coincide con resultados de la anterior investigación realizada en el año 2017, siendo en promedio 42, el valor hallado en la indagación previa. Puede valorarse los resultados de este componente en la siguiente gráfica:

\section{Gráfica 1.}

Componente cognitivo de la actitud docente hacia las TIC

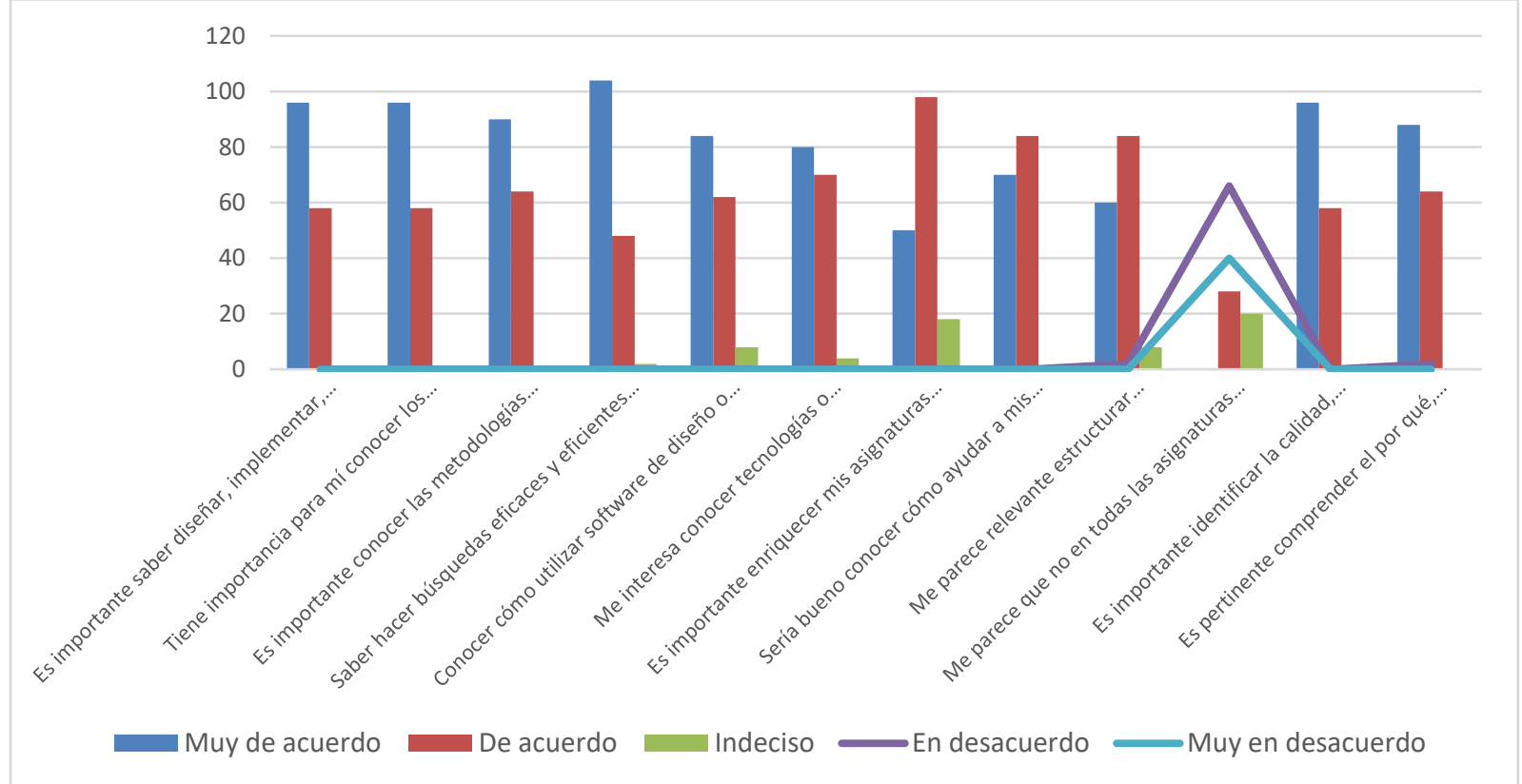

Fuente: Elaboración propia a partir de la encuesta "Actitudes, usos e intereses en las TIC", 2010. 
Con respecto al componente afectivo, se halla actualmente un valor promedio de 48 , lo cual indica una actitud positiva en favor de las TIC. Este es el componente más relevante de las actitudes, puesto que implica sentimientos a favor o en contra para hacer uso de estas herramientas.

Dentro de la encuesta, existen tres preguntas que aportan para la identificación de actitudes negativas en este ámbito, se halló que un $25 \%$ de los docentes sienten desconfianza y temor frente al uso de las TIC, un $84 \%$ considera que es importante la relación presencial con el estudiante para obtener mejor aprendizaje, y 32\% siente confusión con la variedad de información y su calidad en internet. No obstante, a pesar de estos resultados, la valoración global del componente afectivo, es positiva.

Puede valorarse, los diferentes ítems y su apreciación, en la siguiente gráfica.

\section{Gráfica 2.}

Componente afectivo de las actitudes docentes hacia el uso de las TIC

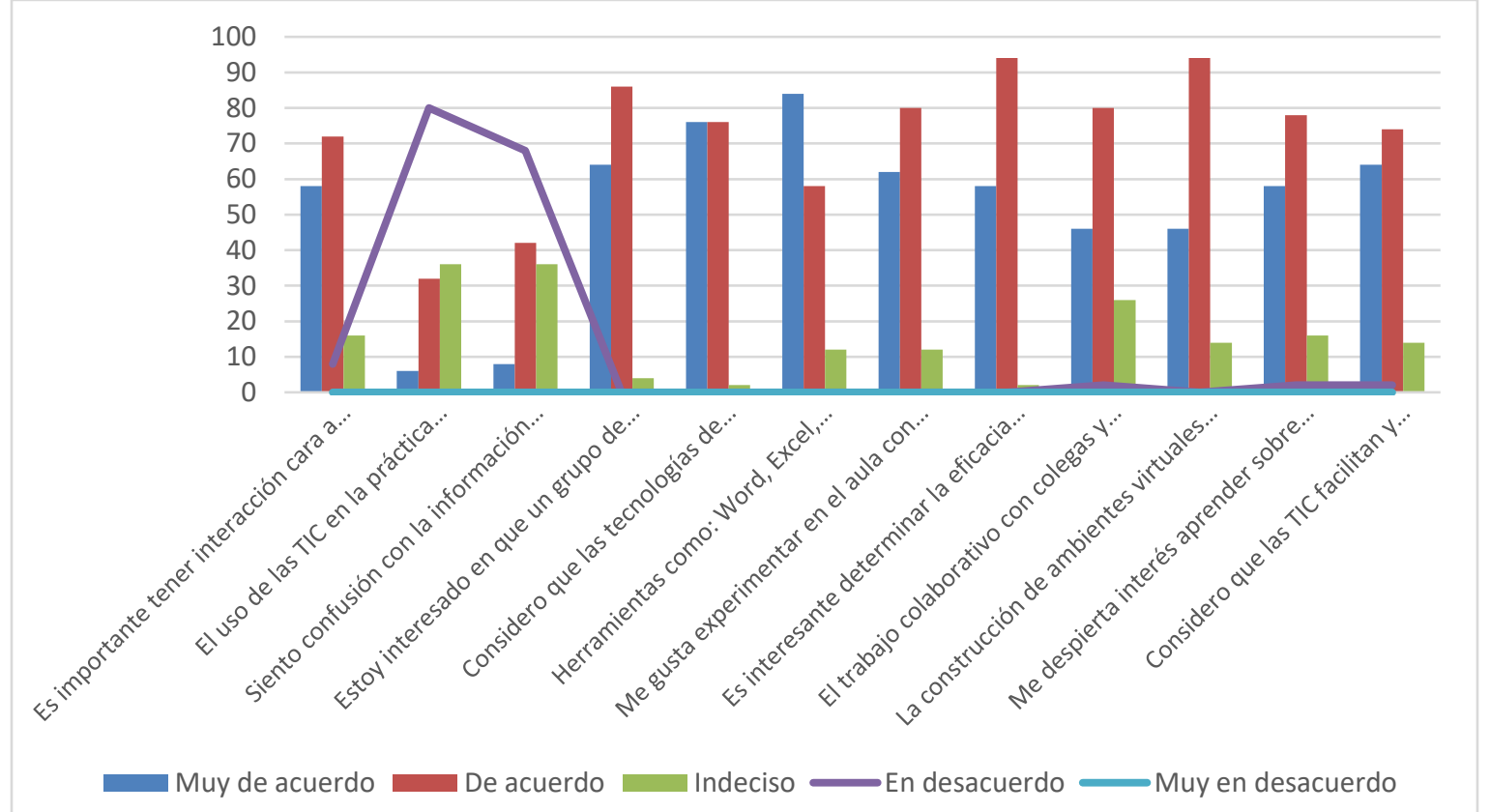

Fuente: Elaboración propia a partir de la encuesta "Actitudes, usos e intereses en las TIC", 2010.

Los resultados hallados en este componente en la actual investigación, discrepan de los obtenidos en 2017 , puesto que se había obtenido un valor promedio de 35 , lo cual se enmarca en una actitud indecisa o medianamente negativa.

Así mismo, se indagó en el componente pedagógico conductual de las actitudes, encontrando que actualmente los docentes otorgan en promedio a este, un valor de 51 , lo cual pone en evidencia estar muy de acuerdo, no sólo con la utilización de herramientas que plantean el desarrollo de competencias de profundización y generación de conocimiento, sino un interés 
encaminado a tomar liderazgo en formar comunidades de aprendizaje virtual. Estos datos son muy diferentes a la anterior evaluación en la cual los docentes puntuaban esta subcategoría con promedio de 40 . Los datos actuales pueden evidenciarse en la gráfica siguiente.

\section{Gráfica 3.}

Componente conductual de las actitudes docentes hacia el uso de las TIC

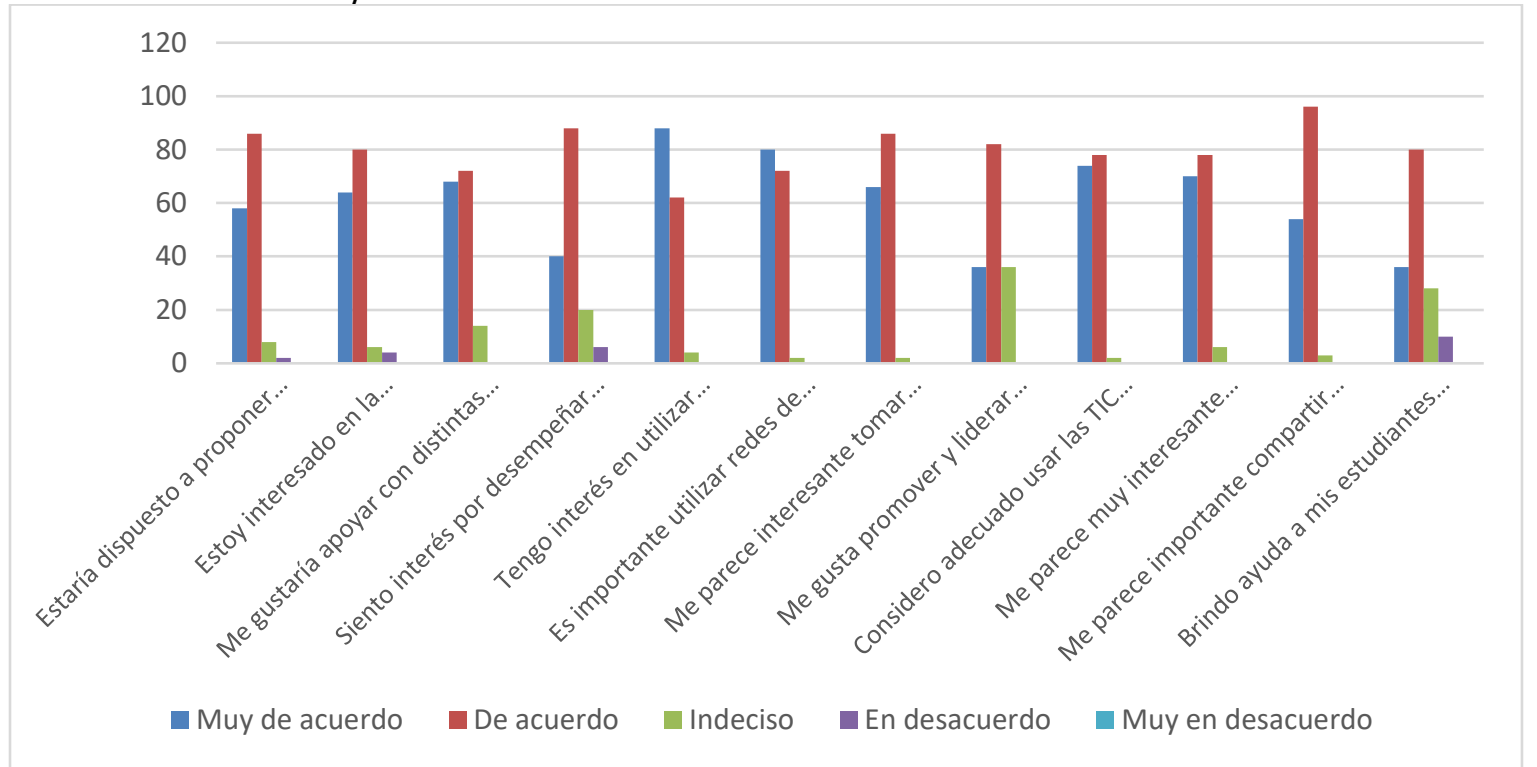

Fuente: Elaboración propia a partir de la encuesta "Actitudes, usos e intereses en las TIC" (2010)

Acorde a los datos hallados, se analiza que dentro de los tres componentes pedagógicos que integran la actitud, se configura una actitud en general positiva para el uso de las TIC en la actualidad.

\section{Frecuencia de uso, experticia e interés de aprendizaje en las TIC}

De otra parte, se evaluó de manera diferenciada la frecuencia de uso e interés de aprendizaje por las Tecnologías de la Información (TI) y, la experticia e interés por las Tecnologías de la Comunicación (TC); las primeras incluyen herramientas que permiten digitalizar, almacenar, procesar y transmitir contenidos digitales; las segundas hacen referencia a los medios o canales que hacen posible la interacción comunicativa.

Es relevante hacer ver que, en medio de la pandemia, la universidad en la cual se desarrolló el estudio diseñó un programa educativo virtual para todos los docentes. En este participó la totalidad de profesores de la facultad evaluada; la investigación se realizó tres semanas posteriores al inicio de la capacitación, la cual se orientó en temas relacionados con la aplicación de las tecnologías de información y comunicación en los cursos y el manejo de la plataforma virtual institucional.

Respecto a la frecuencia de uso de Tecnologías de Información en las prácticas pedagógicas de los docentes, se halló un incrementó en el evento actual. Lo anterior y la diferencia entre 
periodos evaluados, se evidencia en la siguiente tabla:

Tabla 1.

Frecuencia de uso e interés de aprendizaje en las Tecnologías de Información de los docentes universitarios de la Facultad de Ciencias de la Salud

\begin{tabular}{lllllll}
\hline TIC UTILIZADA & $\begin{array}{l}\text { AÑO } \\
\text { EVALU }\end{array}$ & $\begin{array}{l}\text { NUNCA } \\
\text { O CASI }\end{array}$ & $\begin{array}{l}\text { OCASION } \\
\text { ALMENTE }\end{array}$ & $\begin{array}{l}\text { CASI } \\
\text { SIEMPR }\end{array}$ & $\begin{array}{l}\text { SIEMP } \\
\text { RE }\end{array}$ & $\begin{array}{l}\text { INTERÉS DE } \\
\text { FORMACIÓN }\end{array}$ \\
& 2017 & $32 \%$ & $12 \%$ & $28 \%$ & $28 \%$ & Algo interesado \\
Procesador de texto & 2020 & $0 \%$ & $2.6 \%$ & $38 \%$ & $59.4 \%$ & Algo interesado \\
& 2017 & $40 \%$ & $12 \%$ & $32 \%$ & $16 \%$ & Algo interesado \\
Procesador gráfico (Paint, & 2020 & $9 \%$ & $42 \%$ & $22 \%$ & $27 \%$ & Muy interesado \\
Photoshop, Corel) & 2017 & $16 \%$ & $44 \%$ & $20 \%$ & $20 \%$ & Algo interesado \\
Hojas de cálculo (Excel) & 2020 & $0 \%$ & $30 \%$ & $34 \%$ & $36 \%$ & Muy interesado \\
& 2017 & $36 \%$ & $20 \%$ & $36 \%$ & $8 \%$ & Algo interesado \\
Procesamiento de audio y & 2020 & $32 \%$ & $39 \%$ & $14 \%$ & $15 \%$ & Muy interesado \\
video (Windows, Movie & & & & & & \\
Maker...) & 2017 & $16 \%$ & $32 \%$ & $44 \%$ & $8 \%$ & Algo interesado \\
Video tutoriales & 2020 & $13 \%$ & $43 \%$ & $29 \%$ & $16 \%$ & Muy interesado \\
& 2017 & $0 \%$ & $4 \%$ & $28 \%$ & $68 \%$ & Muy interesado \\
$\begin{array}{l}\text { Presentaciones multimedia } \\
\text { (PowerPoint) }\end{array}$ & 2020 & $0 \%$ & $21 \%$ & $43 \%$ & $36 \%$ & Algo interesado \\
Paquetes estadísticos (SPSS, & 2017 & $51 \%$ & $40 \%$ & $6 \%$ & $3 \%$ & Algo interesado \\
etc.) & 2020 & $46 \%$ & $31 \%$ & $13 \%$ & $10 \%$ & Algo interesado \\
$\begin{array}{l}\text { Fuentes digitales de } \\
\text { información (motores de }\end{array}$ & 2017 & $16 \%$ & $8 \%$ & $52 \%$ & $24 \%$ & Algo interesado \\
búsqueda, etc.) & 2020 & $0 \%$ & $32 \%$ & $35 \%$ & $33 \%$ & Muy interesado \\
\hline
\end{tabular}

Fuente: Elaboración propia a partir de la encuesta "Actitudes, usos e intereses en las TIC" (2010)

En la evaluación actual, emerge el alto y mediano uso de herramientas que permiten mayor interacción en la modalidad virtual, las cuales se usaban muy escasamente durante el periodo anterior. A continuación, se describen las herramientas de alto y mediano uso:

Alto uso: procesadores de texto, hojas de cálculo, fuentes digitales de información como motores de búsqueda y presentaciones multimedia y video tutoriales

Mediano uso: procesadores gráficos como Paint

Dentro de aquellas descritas como de uso escaso, se hallaron el procesamiento de audio y video, y paquetes estadísticos, sin embargo, refieren estar muy interesados en el aprendizaje de las mismas.

En relación al nivel de experticia en el uso de las tecnologías de comunicación, se obtienen resultados muy heterogéneos, es decir, mientras algunos docentes consideran poseer un nivel alto de experticia en algunas herramientas, otros consideran no tener ninguna habilidad en ellas. No obstante, de acuerdo con los resultados, más de la mitad de los docentes encuestados (87\%), maneja un nivel de experticia alto, como se relaciona a continuación.

Nivel avanzado: Correo electrónico, chat (WhatsApp), conferencias con video y audio, creación 
de lecciones interactivas, construcción de objetos de estudio con herramientas que apoyan las labores educativas ( $71 \%$ de docentes en promedio en estas TC), siendo estas últimas, tecnologías del nivel III, consideradas como creación de conocimientos.

Nivel intermedio: tomar parte activa en comunidades de aprendizaje, participación en una comunidad virtual, los foros de discusión ( $87 \%$ en promedio en estas TC)

Nivel básico: Blogs. Un 56\% de docentes, refiere no conocerlo, pero expresan poseer un alto grado de interés por aprender. El porcentaje restante refiere tener un nivel básico en su manejo.

Alto grado de desconocimiento: Wikis. Esta TC es desconocida por el $90 \%$ de los docentes. Así mismo, se halla un porcentaje de $72 \%$ de desconocimiento en el manejo de portafolios digitales.

Dos de las herramientas más utilizadas por el cuerpo docente encuestado son las presentaciones multimedia y video tutoriales, lo cual guarda relación con la experticia señalada, dado que más de la mitad de los profesores indicaron contar con un nivel superior en el dominio de estas herramientas.

\section{Obstáculos para el uso y apropiación de las TIC en las prácticas pedagógicas}

Un alto porcentaje de docentes (68\%) percibe que la falta de apoyo conceptual y tecnológico para integrar las TIC en los cursos, y la ausencia de formación especializada en las diversas áreas y herramientas informáticas, ha sido uno de los obstáculos para la utilización de las TIC en sus prácticas pedagógicas.

\section{Discusión y conclusiones}

La pandemia actual, se presentó como un punto de inflexión o de giro que ocasionó en el docente repensar sus prácticas pedagógicas mediáticas y buscar estrategias para pasar de la modalidad presencial a la modalidad virtual exclusiva en la educación. Lo anterior, conllevó a realizar cambios en los procesos educativos, ajustes en las subjetividades y actuaciones docentes frente al uso y formación en las TIC, como todo un trasegar de programaciones institucionales para capacitar de manera urgente a los docentes, quienes debieron generar y compartir contenidos de sus cursos a partir de nuevas aplicaciones, herramientas y canales de comunicación virtual, flexibilizar sus prácticas de enseñanza y entender, en medio de todo este proceso, la diversidad de contextos en los cuales se inserta el estudiante, para ofertar una educación coherente al mismo.

Bajo este panorama subyacen varias aristas del tema, pero en forma estricta vamos a hacer alusión a lo planteado en los objetivos del estudio. Así las cosas, se identifica en el actual momento histórico, actitudes favorables de los docentes frente a la inclusión de las TIC en sus 
prácticas pedagógicas, y aun cuando en los programas de salud se posea una percepción tal vez sobrevalorada, como refiere Núñez et., ál (2019), de la necesidad de interactuar cara a cara entre estudiantes y docentes para llevar a cabo procesos de enseñanza y aprendizaje, estos resultados revelan cambios notables frente a datos del periodo anterior 2017, así como estudios que evidencian actitudes negativas de los docentes para avanzar en el uso de las TIC (Rivera, Fernández, Guzmán y Pulido, 2017).

Respecto al uso de las tecnologías de información, actualmente los resultados divergen del estudio realizado en el periodo anterior, como de los hallazgos de Padilla (2018) y de Said (2012), en los cuales identifican mayor uso de herramientas básicas sin pretensión de innovar o de replantear prácticas pedagógicas, lo cual pone de relieve que en este evento del Covid-19, las características del contexto y del acontecimiento, han sido un punto de cambio para iniciar el uso de tecnologías de información con nivel de profundización o creación de conocimiento, definidos de esta forma por la Unesco.

A partir de las necesidades del contexto y de la normatividad emanada por las directivas institucionales durante la pandemia y el confinamiento social obligatorio prolongado, los docentes han realizado autoaprendizajes, se han acogido a programas de capacitación institucional, y realizan propuestas como "conocer nuevas plataformas educativas donde el estudiante pueda interactuar con ellas y hacer más amena la clase", "aportar a los microcurrículos elementos de la virtualidad que hagan los cursos amigables e interactivos", entre otros, lo cual permite valorar un panorama de apertura, no solo al uso frecuente de las TIC, sino a las diversas formas que promuevan la convergencia entre las tecnologías y un modelo pedagógico en el que su inclusión conlleve a aquello que Larrosa (2009) propone como una experiencia educativa que marca huellas en el estudiante.

Todo lo anterior, amerita una mirada de análisis para ver de un lado las necesidades del docente, que ha tenido que emprender una carrera de aprendizaje sobre la marcha en el uso de la TIC, dando un giro al desarrollo de las actividades académicas tradicionales, así como a los espacios de interacción, pero además a un nuevo modelo pedagógico que ostente por el análisis crítico, la interacción en doble vía, la permanente evaluación de los procesos y retroalimentación de los mismos, con fines de mantener la calidad educativa en un contexto de virtualidad que privilegie la pertinencia de los procesos y las realidades situadas.

Como refiere Suárez (2020), este cambio trascendental, requiere una reforma no solo institucional, sino de las políticas formativas universitarias, puesto que se debe atender las necesidades tanto de estudiantes como de docentes, infraestructuras y equipos para continuar los procesos de enseñanza y aprendizaje e innovar nuevos programas y formas de interactuar en la virtualidad. 


\section{Referencias}

Álvarez, S., Cuéllar, C., López, B., Adrada, C., Anguiano, R., Bueno, A., Comas, I., y Gómez, S. (2011). Actitudes de los profesores ante la integración de las TIC en la práctica docente. Estudio de un grupo de la Universidad de Valladolid. Revista Electrónica de Tecnología Educativa, (35), 149-160. https://doi.org/10.21556/edutec.2011.35.416

Araya, V., y Orellana, X. A. (2018). Representaciones de docentes universitarios respecto de las TIC en la acción práctica. Revista de estudios y experiencias en Educación, 2(3Esp), 45-58. https://doi.org/10.21703/rexe.Especial3_201845584

Ávila, W. (2013). Hacia una reflexión histórica de las TIC. Hallazgos, 10(19), 213-233. https://www.redalyc.org/articulo.oa?id=4138/413835217013

Cabero, J. (2015). Reflexiones educativas sobre las tecnologías de la información y la comunicación (TIC). Revista Tecnología, Ciencia y Educación, (1), 19-27. https://www.researchgate.net/publication/278455870_Reflexiones_educativas_sobre_las_ tecnologias_de_la_informacion_y_la_comunicacion_TIC/citation/download

Cariaga, R. (2018). Experiencias en el uso de las TIC. Análisis de relatos docentes. Ciencia, Docencia y Tecnología, 29(56), 131-155. https://doi.org/10.33255/2956/332

Castells, M. (1996). La era de la información. Economía, sociedad y cultura. Siglo XXI.

Castells, M. (2008). Materiales para una teoría exploratoria de la sociedad de redes. The British Journal of Sociology, 51(1), 5-24. https://doi.org/10.1111/j.1468-4446.2000.00005.x

Cázares, I., y Urbiola, A. (2015). Estudiantes universitarios, en el uso de las herramientas tecnológicas ¿activos o pasivos? Educación y Humanismo, 17(28), 15-28. http://dx.doi.org/10.17081/eduhum.17.28.1163

Cejas-León, R., y Navío-Gámez, A. (2020). Sobre la formación tecnopedagógica del profesorado. La visión de los expertos y formadores. Revista Iberoamericana de Educación Superior, 11(31), 150-164. https://doi.org/10.22201/iisue.20072872e.2020.31.711

Colina, A., y Túa, J. (2018). Apropiación de las TIC en la docencia universitaria: Statu quo ante un panorama complejo. Revista Espacios, 39(43), 1-21. http://www.revistaespacios.com/a18v39n43/a18v39n43p21.pdf

Departamento Nacional de Planeación. (2018,15 de diciembre). Evaluación de los programas Vive Digital para la gente financiado con recursos del Fondo de Tecnologías de la Información y Comunicaciones (FONTIC). https://colaboracion.dnp.gov.co/CDT/Prensa/EstudioFONTIC.pdf 
Departamento Nacional de Planeación. (2020, 31 de marzo). Documento Conpes 3988. https://www.dnp.gov.co/CONPES/documentos-conpes/Paginas/documentos-conpes.aspx

Dussel, I. (2015). Incorporación con sentido pedagógico de TIC en la formación docente de los países del Mercosur. Teseo.

Echeverri, J. (2014). Percepción de los profesores de las carreras del área de la salud de la Universidad CES, Medellín, sobre el uso de las nuevas tecnologías de la Información y Comunicación en los procesos de enseñanza. [tesis de maestría, Universidad San Buenaventura].

Biblioteca

Digital.

http://bibliotecadigital.usb.edu.co:8080/handle/10819/2282

Ehuletche, A., Lado, S., Atlante, M., y Malbernat, L. (2018). Competencias para el uso de tecnologías educativas de docentes del nivel superior. Análisis longitudinal del periodo 20122017 en América Latina. Virtualidad, educación y Ciencia, 17(9), 9-21.

García, A., Ulloa, M., y Córdoba, M. (2020). La era digital y la deshumanización. Reidocrea, (9), 11-20. https://www.ugr.es/ reidocrea/9-2.pdf

Herdina, P., \& Jessner, U. (2002). A dynamic model of multilingualism: Perspectives of change in Psycholinguistics. Multilingual Matters.

Katz, R. (2009). El papel de las TIC en el desarrollo. Propuesta de América Latina a los retos económicos actuales. Ariel, S.A.

Larrosa, J. (2009). Experiencia y alteridad en educación. En C. Skliar, \& J. Larrosa, Experiencia y alteridad en educación (p. 25). Homo Sapiens Ediciones.

Lucumí, P., y González, M. (2015). El ambiente digital en la comunicación, la actitud y las estrategias pedagógicas. Tecné, Episteme y Didaxis, 37(37), 109-129. https://doi.org/10.17227/01213814.37ted109.129

MEN. (2017, 12 de octubre). Plan Nacional Decenal de Educación 2016-2026. El camino hacia la calidad y la equidad. Ministerio de Educación Nacional. http://www.plandecenal.edu.co/cms/media/herramientas/PNDE\%20FINAL_ISBN\%20web. pdf

Ministerio de Salud y Protección Social. (12 de marzo de 2020). Resolución Nº 385 del 12 de marzo de 2020. Bogotá, Colombia.

Núñez, C., Gaviria, J., Tobón, S., Guzmán, C., y Herrera, S. (2019). La práctica docente mediada por TIC: una construcción de significados. Espacios, 40(5), 4. https://revistaespacios.com/a19v40n05/a19v40n05p04.pdf 
OPS-OMS. (2020). Actualización epidemiológica Enfermedad por Coronavirus (COVID 19). Washington, D.C.: OPS.

Padilla, S. (2018). Usos y actitudes de los formadores de docentes ante las TIC. Entre lo recomendable y la realidad de las aulas. Apertura, 10(1) ,132-148. http://dx.doi.org/10.32870/ap.v10n1.1107

Congreso de la República de Colombia. (2009, 30 de julio). Ley 1341 de 2009. https://www.mintic.gov.co/portal/inicio/3707:Ley-1341-de-2009

Rivera, L., Fernández, K., Guzmán, F., y Pulido, J. (2017). La aceptación de las TIC por profesorado universitario: conocimiento, actitud y practicidad. Educare, 21(3), 1-18. http://dx.doi.org/10.15359/ree.21-3.6

Said, E. (2012). O uso das TIC por docentes de cenários de ensino superior na Colômbia. Convergencia. Revista de Ciencias Sociales, 19(58), 133-155. https://www.redalyc.org/articulo.oa?id=10520680007

Salazar, D. (2015). Las TIC como desarrollo personal y social. Desbordes, 6, 151- 153. https://hemeroteca.unad.edu.co/index.php/desbordes/article/view/1876/2091

Sánchez, D., y Robles, M. (2016). Riesgos y potencialidades de la era digital para la infancia y la adolescencia. Educación y Humanismo, 18(31), 186-204. https://doi.org/10.17081/eduhum.18.31.1374

Sánchez, M., García, J., Steffens, E., y Hernández, H. (2019). Estrategias Pedagógicas en Procesos de Enseñanza y Aprendizaje en la Educación Superior incluyendo Tecnologías de la Información y las Comunicaciones. Información Tecnológica, 30(3), 277-286. https://doi.org/10.4067/S0718-07642019000300277

Suárez, N. (2020). Formación docente universitaria y crisis sanitaria Covid-19. Ecuador. CienciAmérica, 9(2). http://dx.doi.org/10.33210/ca.v9i2.299

Tapia, H. (2018). Actitud hacia las TIC y hacia su integración didáctica en la formación inicial docente. Actualidades Investigativas en Educación, 18(3), 1-29. http://dx.doi.org/10.15517/aie.v18i3.34437

Tejada, J., y Pozos, K. (2018). Nuevos escenarios y competencias digitales docentes: hacia la profesionalización docente con TIC. Revista de curriculum y formación del profesorado, 22(1), 25-51. https://recyt.fecyt.es/index.php/profesorado/issue/view/3262

Tello, I., y Cascales, M. (2015). Las TIC y las necesidades específicas de apoyo educativo: análisis de las competencias TIC en los docentes. Revista Iberoamericana de Educación a Distancia, 
18(2), 355-383.

Tobón, M., Arbeláez, M., Falcón, M. y Bedoya, J. (2010). La formación docente al incorporar las TIC en los procesos de enseñanza y aprendizaje. https://www.adeepra.org.ar/congresos/Congreso\%20IBEROAMERICANO/TICEDUCACION/ R1998Tobon.pdf

Unesco. (2019, 20 de marzo). Marco de competencias de los docentes en materia de TIC. http://eduteka.icesi.edu.co/pdfdir/unesco-competencias-tic-docentes-version-3-2019.pdf

UNESCO. (2020, 15 de junio). Iniciativas de aprendizaje a distancia basadas en uso de internet y otros medios de comunicación. https://www.siteal.iiep.unesco.org/respuestas_educativas_covid_19

Vesga, L., y Vesga, J. (2012). Los docentes frente a la incorporación de las TIC en el escenario escolar. Revista de historia de educación latinoamericana, 14(19), 247-263. http://dx.doi.org/10.9757/Rhela.19.11 